\title{
EVALUACIÓN QUÍMICO BROMATOLÓGICA DE LAS VARIEDADES YURAC LLAJUM, QELLO LLAJUM Y YURAC CHECCHE DE Smallanthus sonchifolius (POEPP \& ENDL).H. ROBINSON (YACÓN) PROCEDENTE DE PUNO
}

\author{
Chemical and bromatological evaluation of varieties Yurac llajum, Qello llajum y Yurac chec- \\ che of Smallanthus sonchifolius (Poepp \& Endl) H. Robinson (yacón) from Puno
}

Rubén Ramos Zapana y Gladys Arias Arroyo

Laboratorio de Bromatología, Facultad de Farmacia y Bioquímica. Universidad Nacional Mayor de San Marcos

\section{RESUMEN}

Las variedades Yurac llajum, Qello llajum y Yurac checche de la especie Smallanthus sonchifolius (Poepp \& Endl) (Yacón), procedente de la provincia de Sandia del Departamento de Puno, se desarrollan entre 1500 a 3000 msnm. Conocidas como “yacón”, “yakuma”, "llaqón”, "llacun” o "llacuma” en quechua; en aymara "aricoma” o "aricuma“; en español “Yacón”, “Jacón”, “llacón”, “arboloco”, “Puhe”, “jicama”, “jíquima”, “jikima” o “jiquimilla”. Estas raíces tuberosas de sabor dulce y refrescante, de aspecto granuloso, blanco amarillento y forma alargada presentan en promedio por planta, un peso de $248,70 \mathrm{~g}$, una longitud de $17,16 \mathrm{~cm}, 15$ raíces y $6,20 \mathrm{~cm}$ de diámetro de raíz. Presentan un contenido de agua de 80,83 a 87,05 gramos por ciento. En muestra seca: proteína total 2,76 a 2,86; ceniza 2,78 a 2,92; extracto etéreo o,27 a o,29; fibra cruda 4,07 a 4,34; carbohidratos de 89,53 a 90,86; azúcares reductores directos 7,04 a 8,14; azúcares reductores totales 25,33 a 27,65 y acidez total de 0,27 a 0,32; todo considerado en gramos por ciento.

Palabras clave: Smallanthus sonchifolius, yacón, Yurac llajum, Qello llajum y Yurac Checche.

\section{SUMMARY}

The varieties Yurac llajum, Qello llajum y Yurac Checche of the species Smallanthus sonchifolius (Poepp \& Endl) (Yacón), coming from the province of Sandia, Department of Puno, it is developed from 1500 to 3000 msnm, also are well-known like "yacón”, “yakuma”, "llaqón”, “ llacun” and "llacuma” in quechua language; "aricoma” and "aricuma" in aymara language; "Yacón”, “Jacón”, "llacón”, “arboloco”, "Puhe”, “jicama”, “jíquima”, “jikima” y "jiquimilla” in Spanish. These tuberose roots of sweet and refrescante (refreshing?) flavor, of granulate aspect, white yellowish and forms extended show an average of: weight of $248,70 \mathrm{~g}$ by plant, a length of $17,16 \mathrm{~cm}, 15$ roots by plant, and 6,20 cm of diameter of root by plant. It presents a content of water of 80,83 to 87,05 ; total protein 2,76 to 2,86 ; ash 2,78 to 2,92 ; etéreo extract o,27 to o,29; crude fiber 4,07 to 4,34; carbohydrates 89,53 to 9o,86; sugar direct reducers 7,04 to 8,14; sugar total reducers 25,33 to 27,65 and total acidity 0,27 a 0,32 ; all considered in grams percent.

Keywords: Smallanthus sonchifolius, yacón, Yurac llajum, Qello llajum y Yurac Checche.

\section{INTRODUCCIÓN}

$\mathrm{E}$ l yacón es una planta perenne que se encuentra en Ecuador, Bolivia, Colombia, Venezuela, Argentina, Nueva Zelanda, Japón y Brasil. En el Perú se produce en Cajamarca, Huánuco, Cusco, Abancay, Amazonas, y Puno. El género Smallanthus comprende 21 especies, de las cuales siete han sido encontradas en el Perú (1) y, según Meza ${ }^{(2)}$, se conocen cinco variedades de yacón; conocido también como llakwash en el norte del Perú (Incawasi, Ferreñafe, Lambayeque); los nativos bilingües muchik dicen que significa alimento aguanoso. En aymara se le conoce como aricoma o aricuma y en quechua yacón, llaqón, llacum, yakuma o llacuma

En la frontera de Bolivia con Brasil, Argentina y Paraguay se le conoce como ipio, en idioma chiriguano. En Ecuador, el nombre común es jicama, con derivaciones como chicama, shicama, jiquima y jiquimilla; se considera que estos términos son una derivación de xicama, palabra mexicana aplicada a especies del genero Pachyrhizus. En Colombia y Venezuela se le conoce como jiquima y jiquimilla. Los nombres comunes que presenta el yacón, son abundantes por lo que es necesario indicar el nombre de esta raíz. 
El yacón tradicionalmente se consume como fruta fresca o deshidratada. También se consume, aunque de manera ocasional y solamente en algunas localidades, en forma de jalea y de chicha. Al estado fresco es un buen rehidratante debido a su alto contenido de agua. Además, puede prevenir la fatiga y los calambres por su alto contenido de potasio. Tal vez por ello, los campesinos lo consumen durante largas caminatas, pudiendo llegar a consumir cada persona entre 500 a $1000 \mathrm{~g}$ diarios. Este nivel de consumo no produce efectos tóxicos o nocivos.

Esta especie es cultivada con diversos fines: $83,47 \%$ se destina a la venta (antes en trueques); 4,30\% se consume y el $2,53 \%$ se destina a la alimentación de algunos animales.

Si bien el yacón es un alimento, se ha reportado su uso medicinal. En Contumaza (Cajamarca), se le considera antirraquítico. En la medicina andina las raíces son consideradas alimentos frescos y empleados desde épocas muy antiguas como remedio para afecciones renales y hepáticas.

Casi todas las variedades fueron y están siendo estudiadas desde el punto de vista botánico, bromatológico y químico en la Universidad Nacional Agraria La Molina, el Centro Internacional de la Papa y otras universidades del país. Sin embargo, de las variedades de la raíz tuberosa de la provincia de Sandia - Puno, hasta la fecha se tienen como antecedentes sólo estudios agronómicos, mas no estudios químico-bromatológicos. Además, debido a que recientemente se han empezado a descubrir y difundir algunas de sus propiedades promisorias, se ha generado en la población y en la industria alimentaria (como alimento funcional) un creciente interés por este producto. Es por todas estas razones que se ha planteado en el presente proyecto el estudio de las características físicas y un análisis químico-bromatológico, para ampliar el conocimiento sistematizado y fomentar mejor aprovechamiento de sus cualidades.

\section{MATERIALES Y MÉTODOS}

Las muestras en estudio se recolectaron de la provincia de Sandia, ubicada en la zona nor-oriental del departamento de Puno.

\section{Recolección, transporte y conservación de la muestra}

Las muestras se recolectaron en bolsas de polietileno, correspondiente a la campaña agrícola 2004-2005. El método que se utilizó fue estadístico o aleatorio.

\section{Preparación de la muestra}

Para realizar el estudio químicobromatológico, las variedades escogidas de yacón (raíz) provenientes de la provincia de Sandia fueron sometidas a proceso de liofilización. Se tomaron 15 a 20 raíces, las que fueron divididas longitudinalmente en dos partes y luego cortadas

Tabla 1. Características organolépticas de la raíz de las variedades Yurac llajum, Qello llajum y Yurac checche de Smallanthus sonchifolius (Poepp \& Endl)(Yacón)

\begin{tabular}{cccc}
\hline Características & Yurac & Qello & Yurac \\
llajum & $\begin{array}{c}\text { llajum } \\
\text { checche }\end{array}$ & Granuloso \\
Aspecto & Granuloso & Granuloso & Blanco grisáseo \\
Color & Blanquecino & Blanco amarillento & Insípido \\
Olor & Insípido & Insípido & Dulce \\
Sabor & Dulce & Dulce & \\
\hline
\end{tabular}

Tabla 2. Características morfológicas de la raíz de las variedades Yurac llajum, Qello llajum y Yurac checche de Smallanthus sonchifolius (Poepp \& Endl)(Yacón)

\begin{tabular}{|c|c|c|c|}
\hline Características & $\begin{array}{l}\text { Yurac } \\
\text { llajum }\end{array}$ & $\begin{array}{l}\text { Qello } \\
\text { llajum }\end{array}$ & $\begin{array}{l}\text { Yurac } \\
\text { checche }\end{array}$ \\
\hline $\begin{array}{l}\text { Peso promedio de raíz por } \\
\text { planta (g) }\end{array}$ & 269,30 & 248,7 & 207,20 \\
\hline $\begin{array}{l}\text { Longitud promedio de raíz } \\
\text { por planta }(\mathrm{cm})\end{array}$ & 13,80 & 17,16 & 21,62 \\
\hline $\begin{array}{l}\text { Número promedio de } \\
\text { raíces por planta }\end{array}$ & 12 & 15 & 14 \\
\hline $\begin{array}{l}\text { Diámetro promedio de raíz } \\
\text { por planta }(\mathrm{cm})\end{array}$ & 7,35 & 6,2 & 5,80 \\
\hline Forma & redondeada & alargada & alargada \\
\hline Color de la cascara & $\begin{array}{l}\text { rosado } \\
\text { blanco }\end{array}$ & crema amarillo & crema oscuro blanco \\
\hline
\end{tabular}


Tabla 3. Composición química de la raíz de las variedades Yurac llajum, Qello llajum y Yurac Checche de Smallanthus sonchifolius (Poepp \& Endl) (Yacón)

\begin{tabular}{|c|c|c|c|c|c|c|}
\hline & \multicolumn{2}{|c|}{ Yurac llajum } & \multicolumn{2}{|c|}{ Qello llajum } & \multicolumn{2}{|c|}{ Yurac checche } \\
\hline & $\begin{array}{c}\text { Muestra fresca } \\
\text { (g\%) }\end{array}$ & $\begin{array}{c}\text { Muestra seca } \\
(\text { (g\%) }\end{array}$ & $\begin{array}{c}\text { Muestra fresca } \\
\text { (g\%) }\end{array}$ & $\begin{array}{c}\text { Muestra seca } \\
(\mathrm{g} \%)\end{array}$ & $\begin{array}{c}\text { Muestra fresca } \\
\text { (g\%) }\end{array}$ & $\begin{array}{c}\text { Muestra seca } \\
\text { (g\%) }\end{array}$ \\
\hline Agua & 84,63 & $\ldots$ & 87,05 & $\ldots$ & 80.83 & $\ldots$ \\
\hline Extracto etéreo & & 0,27 & & 0,28 & & 0,29 \\
\hline Proteína total * & & 2,76 & & 2,84 & & 2,86 \\
\hline Fibra cruda & & 4,34 & & 4,20 & & 4,07 \\
\hline Ceniza & & 2,78 & & 2,83 & & 2,92 \\
\hline Carbohidratos & & 90,86 & & 89,53 & & 89,85 \\
\hline Acidez total & 0,27 & & 0,32 & & 0,31 & \\
\hline $\begin{array}{l}\text { Azúcares reductores } \\
\text { directos }\end{array}$ & & 7,04 & & 7,65 & & 8,14 \\
\hline $\begin{array}{l}\text { Azúcares reductores } \\
\text { totales }\end{array}$ & & 25,43 & & 25,33 & & 27,65 \\
\hline Valor calórico ${ }^{* *}$ & & 376,91 & & 372,0 & & 373.45 \\
\hline
\end{tabular}

en tajadas de dos a tres mm de espesor, tomándose de siete a diez rodajas por raíz. Se colocaron dentro de la bolsa de polietileno con su peso fresco correspondiente y se guardaron en un congelador a -30 grados Celsius hasta el momento de ser liofilizado en un equipo Virtis a $-20{ }^{\circ} \mathrm{C}$ y a una presión de $50 \mathrm{~mm} \mathrm{Hg}$. El tiempo de secado fue en promedio de tres días, al final de los cuales las muestras fueron pesadas para determinar su contenido en materia seca; luego se molieron en un mortero y se guardaron en frascos de polietileno hasta el momento de ser analizadas.

\section{Determinaciones analíticas}

De acuerdo al método descrito por Hidalgo ${ }^{(3)}$ se procedió a pesar las raíces, medir sus diámetros y determinar, por inspección visual, el número de raíces por planta, la forma y color de la cáscara, y el color de la pulpa.

El contenido de agua, proteínas totales, extracto etéreo, cenizas y fibra cruda fueron determinados utilizando los métodos de la AOAC (4). El factor utilizado para calcular proteína fue 6,25. Los carbohidratos fueron obtenidos por diferencia, es decir sustrayendo de 100 la suma de agua, proteína total, extracto etéreo, cenizas y fibra cruda. Los azúcares reductores directos y totales fueron determinados utilizando el método de Lane y Eynon considerado por la AOAC. Los minerales se analizaron utilizando métodos analíticos por espectrofotometría de absorción atómica en un equipo Perkin-Elmer, excepto el fósforo que fue determinado por el método espectrofotométrico

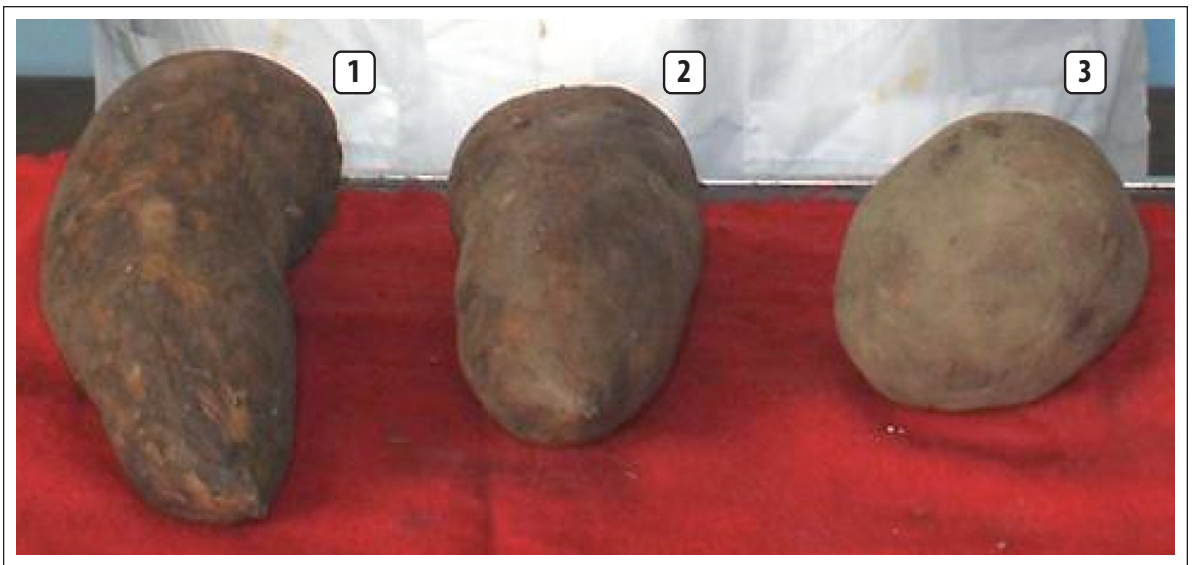

Figura 1. Variedades de yacón de Sandia: 1) Qello llajum 2) Yurac checche 3) Yurac llajum. 
(4). La vitamina $C$ se determinó con el método de titulación 2,6-diclorofenolindofenol (4). La determinación de $\mathrm{pH}$ y acidez total se realizó utilizando los métodos de la AOAC ${ }^{(4)}$.

\section{RESULTADOS}

\section{Características organolépticas de la raíz de yacón}

Las características organolépticas se presentan en la tabla 1.

\section{Características morfológicas de la raíz}

Se presentan en la tabla 2 y se observan las variedades en la figura 1.

\section{DISCUSIÓN}

En cuanto al rendimiento por planta se encontró que la variedad Yurac llajum tiene 269,30 g de raíz por planta, presentando un valor elevado.

El National Research Council (5) indica que los tubérculos del yacón son fusiformes y pueden variar considerablemente en tamaño. El National Research Council ${ }^{(5)}$ señala que los tubérculos del yacón son de tendencia fusiforme y pueden variar considerablemente en tamaño, sabor y en el color de la cáscara que va desde marrón oscuro al purpúreo opaco, e incluso al naranja; internamente presentan un cuerpo carnoso transparente y generalmente pesan de 200 a $500 \mathrm{~g}$, pudiendo llegar hasta los $2 \mathrm{~kg}$.

La longitud encontrada, como se muestra en la tabla 2, varía de 13,80 a $21,62 \mathrm{~cm}$. Al respecto Grau y Rea ${ }^{(6)}$ mencionan que las dimensiones de las raíces pueden llegar a alcanzar valores entre 6 y $25 \mathrm{~cm}$.

El número promedio de raíces que se encontró es de 15 (tabla 2), lo cual está dentro de los valores que reportan Grau y Rea (6), señalando que el número de raíces tuberosas por planta suele estar entre 5 y 40.

Como se muestra en la tabla 2 , se observaron raíces con diámetros de $6,5 \mathrm{~cm}$, valor concordante con lo reportado por Chirinos ${ }^{(7)}$ en raíces procedentes de Cusco.

La forma de las raíces observadas fue bastante variada, habiéndose encontrado entre otras formas alargadas. El color de la cáscara también varía, se han observado matices como amarillo, crema blanco y rosado en las variedades Qello llajum, Yurac checche y Yurac llajum, respectivamente.

El contenido de carbohidratos es alto, el valor promedio en base seca varía entre el 89,53 y 9o,86 g por ciento, tal como se muestra en la tabla 3, valores similares a los encontrados por Lizarraga ${ }^{(8)}, 82$ a $96 \mathrm{~g}$ por ciento en base seca.

En términos generales, los carbohidratos representan alrededor del 90,34\% del peso seco de las raíces recién cosechadas. Entre el 50 y $70 \%$ son fructooligosacaridos (FOS), el resto lo conforman sacarosa, fructosa y glucosa ${ }^{(9,10)}$. Sin embargo, la composición relativa de los diferentes azúcares varían debido a diferentes factores como el cultivo, la época de siembra y cosecha, el tiempo y temperatura en postcosecha ${ }^{(7)}$.

\section{CONCLUSIÓN}

Estas raíces tuberosas de sabor dulce y refrescante, de aspecto granuloso, blanco amarillento y forma alargada presentan un promedio de peso de 241,73 g por planta, una longitud de $17,52 \mathrm{~cm}, 15$ raíces por planta y $6,20 \mathrm{~cm}$ de diámetro de raíz por planta. Presenta en g\%, un contenido de agua de 80,83 a 87,05 . En muestra seca en g\%, proteína total 2,76 a 2,86, cenizas 2,78 a 2,92, extracto etéreo o,27 a 0,29 , fibra cruda 4,07 a 4,34, carbohidratos 89.53 a 9o,86, azúcares reductores directos 7,04 a 8,14, azúcares reductores totales 25,33 a 27,65 y acidez total 0,27 a 0,32 .

\section{REFERENCIAS BIBLIOGRÁFICAS}

1. Brako L, Zarucchi J. Catalogue of the flowering plants and gymnosperms of Peru. Monographs in systematic botany from the Missouri Botanical Garden 45: I-XI; 1993.

2. Meza G. Variedades nativas de yacón (Polymnia sonchifolia) en Cuzco. Universidad Nacional San Antonio de Abad del Cuzco (UNSAAC). Centro de Investigación de Cultivos Andinos (CICA). Cuzco, 1995 .

3. Hidalgo A. Estudio de la influencia del ambiente sobre el color de las hojuelas fritas de dieciseis genotipos de Ipomea batata (L). Lam. [Tesis de Ingeniero en Industrias Alimentarias]. UNALM. Lima, 1990.

4. AOAC. Official Methods of Analysis of the Association of Official Analytical Chemist. 15th ed. Arlington, VA, 1990.

5. National Research Council. Lost crops of the Incas: Little kown plants of the Andes with promise for worldwide 
cultivation. 2da ed. National Academy Press. Washington DC 1998.

6. Grau A \& Rea J. Yacón, Smallanthus sonchifolius (OPEP. \& Endl.) H. Robinson. In: Hermann M \& J Heller. Andean roots and tuber: ahipa, arracacha, naca, yacon. IPGRI. Roma, 1997. p 199-242.

7. Chirinos A. Obtención y caracterización de oligofructanosy la inulina de raíz deyacón (Smallanthus sonchifolia, OPEP. \& Endl. H. Robinson). [Tesis para magíster en industrias alimentarias]. UNALM. Lima, 1999.

8. Lizárraga L, Ortega R, Vargas W, Vidal A. Cultivo del yacón (Polimnia sonchifolia). En resúmenes Curso Pre-congreso - IX Congreso Internacional de cultivos andinos. Cuzco, 1997.

9. Nieto C. Estudios agronómicos y bromatológicos en jicama. Archivos Latinoamericanos de Nutrición, 1991.
XLI (2).

10. Collazos C. La composición de alimentos de mayor consumo en el Perú. 6ta ed. Ministerio de Salud - Instituto Nacional de Nutrición. Lima, 1993.

Manuscrito recibido el: 15/11/2010

Aceptado para su publicación el: 01/02/2011

\section{Correspondencia:}

Nombre: Gladys Arias Arroyo

Dirección: Jr. Puno 1002 - Lima 1 - Perú.

e-mail: ariasarroyo@gmail.com 